\title{
Facilitated physical activity as a treatment for depressed adults: randomised controlled trial
}

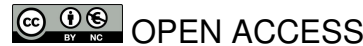

\author{
Melanie Chalder research fellow ${ }^{1}$, Nicola J Wiles senior lecturer ${ }^{1}$, John Campbell professor ${ }^{2}$, Sandra \\ P Hollinghurst senior lecturer ${ }^{1}$, Anne M Haase senior lecturer ${ }^{3}$, Adrian $\mathrm{H}$ Taylor professor ${ }^{4}$, Kenneth \\ R Fox professor ${ }^{3}$, Ceire Costelloe research associate ${ }^{1}$, Aidan Searle research associate ${ }^{1}$, Helen \\ Baxter research associate ${ }^{1}$, Rachel Winder associate research fellow ${ }^{2}$, Christine Wright associate \\ research fellow ${ }^{2}$, Katrina M Turner lecturer ${ }^{1}$, Michael Calnan professor ${ }^{5}$, Deborah A Lawlor \\ professor $^{1}$, Tim J Peters professor ${ }^{6}$, Deborah J Sharp professor ${ }^{1}$, Alan A Montgomery reader ${ }^{1}$, \\ Glyn Lewis professor ${ }^{1}$
}

${ }^{1}$ School of Social and Community Medicine, University of Bristol, Bristol BS8 2BN, UK; ${ }^{2}$ Primary Care Research Group, Peninsula Medical School, Exeter, UK; ${ }^{3}$ School of Policy Studies, University of Bristol; ${ }^{4}$ Sport and Health Sciences, University of Exeter, Exeter; ${ }^{5}$ School of Social Policy, University of Kent, Canterbury, UK; ${ }^{6}$ School of Clinical Sciences, University of Bristol

\begin{abstract}
Objective To investigate the effectiveness of facilitated physical activity as an adjunctive treatment for adults with depression presenting in primary care.

Design Pragmatic, multicentre, two arm parallel randomised controlled trial.

\section{Setting General practices in Bristol and Exeter.}

Participants 361 adults aged 18-69 who had recently consulted their general practitioner with symptoms of depression. All those randomised had a diagnosis of an episode of depression as assessed by the clinical interview schedule-revised and a Beck depression inventory score of 14 or more.

Interventions In addition to usual care, intervention participants were offered up to three face to face sessions and 10 telephone calls with a trained physical activity facilitator over eight months. The intervention was based on theory and aimed to provide individually tailored support and encouragement to engage in physical activity.
\end{abstract}

Main outcome measures The primary outcome was self reported symptoms of depression, assessed with the Beck depression inventory at four months post-randomisation. Secondary outcomes included use of antidepressants and physical activity at the four, eight, and 12 month follow-up points, and symptoms of depression at eight and 12 month follow-up.

Results There was no evidence that participants offered the physical activity intervention reported improvement in mood by the four month follow-up point compared with those in the usual care group; adjusted between group difference in mean Beck depression inventory score
-0.54 (95\% confidence interval -3.06 to $1.99 ; P=0.68$ ). Similarly, there was no evidence that the intervention group reported a change in mood by the eight and 12 month follow-up points. Nor was there evidence that the intervention reduced antidepressant use compared with usual care (adjusted odds ratio $0.63,95 \%$ confidence interval 0.19 to $2.06 ; P=0.44$ ) over the duration of the trial. However, participants allocated to the intervention group reported more physical activity during the follow-up period than those allocated to the usual care group (adjusted odds ratio 2.27, 95\% confidence interval 1.32 to $3.89 ; P=0.003$ ).

Conclusions The addition of a facilitated physical activity intervention to usual care did not improve depression outcome or reduce use of antidepressants compared with usual care alone.

Trial registration Current Controlled Trials ISRCTN16900744.

\section{Introduction}

Depression is one of the most common reasons for consulting a general practitioner within the United Kingdom, and its associated economic burden is considerable. ${ }^{1}$ Although antidepressants are effective, many patients and healthcare professionals would like other options to be available as an alternative or adjunct to drug therapy. ${ }^{2}$ Some evidence ${ }^{3}$ shows that physical activity might be an effective treatment and it has been recommended as part of the latest guidelines on depression from the National Institute for Health and Clinical Excellence. ${ }^{4}$

A Cochrane review ${ }^{3}$ of physical activity interventions and depression suggested a pooled standardised mean difference of 0.82 (95\% confidence interval 0.51 to 1.12 ), based on 23 trials and 907 participants. This evidence on the effectiveness of 
physical activity should be considered with caution, however, owing to several methodological issues. Many of the included trials were small, with even the largest reporting fewer than 50 participants per treatment arm. The majority of trials recruited participants from non-clinical settings, often offering financial or other incentives to those who agreed to participate. Most studies involved relatively short follow-up periods so that evidence for a more sustained benefit is limited, and, finally, few of the interventions designed to increase physical activity were readily applicable to clinical practice. It is therefore difficult to be confident about generalising the pooled results of the meta-analysis ${ }^{3}$ to the population with depression treated by doctors in primary care.

We report the findings of the TREAD (TREAtment of Depression with physical activity) study, ${ }^{5}$ which investigated the effectiveness of a facilitated physical activity intervention in addition to usual care for the treatment of depression in adults presenting in primary care.

\section{Methods}

The TREAD study was a pragmatic, multicentre, two arm parallel randomised controlled trial. Adults presenting with a new episode of depression were randomised to receive either usual care from their general practitioner or usual care plus the TREAD intervention. Full details of the protocol have been published previously ${ }^{5}$ and the results are available in the final report of the trial. ${ }^{6}$

\section{Recruitment of participants and baseline assessment}

Our inclusion criteria were broad as we wanted to recruit adults presenting in primary care with new episodes of depression. However, we excluded those who had failed to respond previously to antidepressants as this group often requires more intensive interventions. We also excluded those aged 70 or more as we thought this older group might have greater physical health problems, making the intervention more difficult to implement. Most of the participants were identified by their general practitioner during routine consultations, although in some practices the electronic patient records were also regularly screened for details of adults with a recent diagnosis of depression to identify any potentially eligible people. The study targeted adults aged 18-69 who either were not taking antidepressants at the time of assessment or had been prescribed antidepressants within four weeks of assessment but had not taken these for at least four weeks before that period. General practitioners excluded those who were unable to complete self administered questionnaires in English; had medical contraindications to physical activity; were being treated for psychosis, bipolar disorder, or major substance misuse; or were pregnant or breast feeding at the time of assessment. At baseline, patients were only eligible to be included in the study if they had a current diagnosis of ICD-10 (international classification of diseases, 10th revision) depressive episode F32 from the computerised clinical interview schedule-revised, ${ }^{78}$ a Beck depression inventory ${ }^{9}$ score of at least 14 , and provided written informed consent. Recruitment for the trial took place between August 2007 and October 2009 in general practices in the south west of England.

\section{Randomisation, concealment, and blinding}

Randomisation was stratified to take account of self reported antidepressant use (yes, no) and was minimised by severity of depression (clinical interview schedule-revised score $\leq 25,26-33$, $\geq 34$ at baseline), recruiting centre (Bristol, Exeter), and self reported level of physical activity $(\leq 1,2-3, \geq 4$ days per week, with at least 30 minutes of moderate or vigorous intensity physical activity). Treatment allocation, concealed from the study researchers using an automated telephone system, was administered remotely and employed a computer generated code. Owing to the nature of the intervention, none of the participants, general practices, clinicians, or researchers performing the outcome assessments could be blinded to treatment allocation.

\section{Follow-up}

Collection of follow-up data took place between August 2008 and October 2010. Participants were asked to provide data at three time points; four, eight, and 12 months post-randomisation, using a self completion questionnaire to eliminate any observer bias. The four month follow-up was chosen as the primary outcome endpoint as it represented the stage in the intervention period at which we expected to observe the largest effect, as well as being an appropriate point at which to review the participants' progress from a clinical perspective. The eight month follow-up broadly coincided with the end of the intervention delivery period,. Data were collected at 12 months to investigate any longer term effects of the intervention on study outcomes. To maximise retention, researchers arranged to meet participants to supervise their data collection at the four and 12 month follow-up points, whenever possible. Participants who were unable to attend these follow-up sessions were contacted by telephone to arrange a further appointment or were sent the questionnaire by post if a face to face session was not possible. Due to restricted resources, eight month follow-up was done solely by postal questionnaire.

\section{Intervention and comparator (usual care)}

Participants in both groups were asked to continue to follow the healthcare advice of their general practitioner for their depression and were therefore free during the trial to access any treatment usually available in primary care, including the use of antidepressants, counselling, referral to "exercise on prescription" schemes, or secondary care mental health services. In addition to this usual care, participants allocated to the intervention group were also offered assistance from a physical activity facilitator.

The intervention was based on theory ${ }^{10}{ }^{11}$ and was designed to provide individually tailored support and encouragement to engage in physical activity (see box for a summary of the key features). A manual ${ }^{6}$ was devised to guide the physical activity facilitators, which described a range of motivational interviewing techniques and behavioural strategies to promote participants' uptake of locally available opportunities for physical activity. The aim was to encourage sustainable activity that could be easily incorporated into the participants' lifestyle. The aspiration was for the participants to engage in moderate or vigorous activity for 150 minutes a week in bouts of at least 10 minutes, but if that seemed unrealistic then the facilitator encouraged any increase in physical activity, whatever the intensity.

The intervention programme comprised an initial hour long face to face assessment session followed by two short telephone contacts, then a further face to face meeting for half an hour. Over the course of 6-8-months, the physical activity facilitator offered up to eight further telephone contacts and one more face to face half hour meeting. The scheduling of sessions was left to the discretion of the physical activity facilitator and the participant, although the intention was to have most sessions 


\author{
Key features of TREAD (TREAtment of Depression with physical activity) intervention \\ Designed to improve long term adherence to physical activity \\ Employed motivational interviewing techniques \\ Individually tailored to reflect participants' needs and preferences \\ Encouraged autonomy and choice of physical activity \\ Provided advice, support, and encouragement \\ Promoted physical activity of all intensities \\ Described in a manual to aid standardisation and dissemination \\ Delivered by a physical activity facilitator \\ Consisted of up to three face to face sessions and 10 telephone calls \\ Lasted about 6-8 months, with most facilitator time in first four months
}

early in the intervention period, to encourage an increase in physical activity, whereas the later sessions were less frequent and designed to maintain any change. The expectation was that at least five sessions, including one face to face meeting, would have been completed well before the primary outcome endpoint at four months post-randomisation. The physical activity facilitators worked part-time, came from a psychology or an exercise science background, and received training in delivering the TREAD intervention and working with participants who had depression. The facilitators were regularly supervised by some of the investigators.

\section{Outcomes}

The primary outcome was the Beck depression inventory (version II) at the four month follow-up. The Beck depression inventory is a self report 21 item scale, with higher scores indicating more severe depression. ${ }^{9}$ The score was treated as both a continuous outcome (range 0-63) and a binary outcome ( $<10$ indicating recovery).

Secondary outcomes included the Beck depression inventory at eight and 12 months post-randomisation and a self reported binary measure of antidepressant use from the four, eight, and 12 month follow-up. Physical activity was measured at all three time points using a self completion seven day recall diary in which participants were asked to record 10 minute bouts of light, moderate, and vigorous physical activity in the week before the assessment. We transformed these data into a single weighted score of MET minutes of physical activity per week (where MET was the metabolic equivalent of the task as a ratio to the basal rate). MET minutes were calculated by multiplying the number of minutes of light activity by 2 , moderate activity by 4.5 , and vigorous activity by $7.5 .^{12}$ Current government guidelines suggest that adults should carry out 150 minutes of moderate or vigorous physical activity per week. ${ }^{13}$ We therefore created a binary variable $(<1000$ MET minutes per week, $\geq 1000$ MET minutes per week) as an approximate guide as to whether participants were meeting this guideline. The recall diary for physical activity was compared with accelerometry data after the four month follow-up in a subset of participants $(n=99)$. Full details of this comparison are in the report of the trial ${ }^{6}$ and indicate that there was reasonable agreement between the two measures, considering that accelerometry and the recall diary measured somewhat different aspects of physical activity. There was, however, greater variability between the accelerometer and recall diary for more vigorous activity. Two further secondary outcomes (health related quality of life $\mathrm{e}^{14}$ and outcome expectations of physical activity ${ }^{15}$ ) were also assessed and analysed; the results are also available in the full trial report. ${ }^{6}$

\section{Statistical analysis}

The statistical analysis and reporting of this trial followed the Consolidated Standards of Reporting Trial guidelines, ${ }^{16}$ and the study protocol was published before the completion of data collection. ${ }^{5}$ All analyses were performed in Stata $11.1,{ }^{17}$ following a prespecified analysis plan approved by the trial steering committee. The primary comparative analyses were conducted using an intention to treat approach without imputation of missing data on outcomes. The primary outcome data (total Beck depression inventory score at four months post-randomisation) were analysed in both continuous and binary form. The continuous outcome was analysed in a linear regression model and is presented as the adjusted difference in mean score between the intervention and usual care groups, taking into account the baseline Beck depression inventory score. The binary outcome was analysed in a logistic regression model and is presented as the adjusted odds ratio of recovery in the intervention group compared with the control group, taking into account the baseline Beck depression inventory score. To account for the variables used for stratification and minimisation in the randomisation process, all models were adjusted for baseline antidepressant use, clinical interview schedule-revised score, recruiting centre, and level of physical activity.

As part of the secondary analyses, the Beck depression inventory score was also considered in a linear repeated measures analysis using data from the four, eight, and 12 month follow-up points. Antidepressant use and physical activity were considered as binary outcomes in a logistic regression model using data from the four month follow-up and also in a logistic repeated measures analysis using data from all three time points. All the analyses using repeated measures examined the possibility of a difference in the effect size over time by introducing a time by group interaction term. The intervention group was coded " 1 " and usual care coded " 0 " in all analyses - therefore a negative difference in means indicates lower Beck depression inventory scores (and better health) in the intervention group, and an odds ratio of more than 1 represents a greater proportion (of individuals recovered, of antidepressants used, or of being physically active) in the intervention group.

Several additional sensitivity analyses of the primary outcome were planned. We investigated the influence of missing data on the results by imputing missing data using multiple imputation chained equation methods (Stata ice procedure version 1.9.5 dated 15 April 2011). ${ }^{17}{ }^{18}$ The imputation model included all those variables associated with "missingness" in the primary outcome at four months post-randomisation as well as additional measures for symptoms of depression (Beck depression inventory score) at later follow-ups. We also used robust estimates in Stata to account for clustering by general practice and a previously published method to account for clustering by 
physical activity facilitator. ${ }^{19}$ As per protocol analyses are likely to be biased, we estimated the complier-average causal effect ${ }^{20}$ using instrumental variable regression techniques, to obtain an unbiased estimate of the effectiveness of the intervention in those who received an "adequate dose" before measurement of the primary outcome at four months post-randomisation. This methodology compares the outcome for those who received an "adequate dose" of the intervention (compliers) with a comparable group of "would be" compliers in the usual care group. For the purposes of this analysis on complier-average causal effect, an "adequate dose" was defined in the analysis plan as having received at least five sessions of the intervention by four months post-randomisation, since this reflected the way in which the intervention was structured. In the instrumental variable approach adopted, randomisation is the instrument, with the assumption that the effect of randomisation on the outcome only operates through its effect on treatment. Finally, two preplanned subgroup analyses for the primary outcome were conducted by including an interaction between treatment allocation and severity of depression or level of physical activity at baseline.

Our sample size calculation indicated that 360 randomised participants would provide $82 \%$ power to detect a third of a standard deviation difference in the Beck depression inventory score as a continuous outcome and $80 \%$ power to detect a $15 \%$ difference in recovery when considering the Beck depression inventory score as a binary outcome, allowing for a $5 \%$ two sided $\alpha$ and a maximum attrition rate of $15 \%$. This sample size calculation was the result of a revision made in the early stages of the trial, following the observation that certain assumptions from the original study protocol did not hold true. Firstly, we had originally expected that $10 \%$ of the sample would have been taking antidepressants at baseline and had intended to exclude them from the main comparative analysis. However, because around $50 \%$ of those randomised into the study were taking antidepressants at baseline, we decided to include everyone in the analysis. This decision was also informed by the results of a trial of internet psychotherapy that did not report any change in antidepressant use in the usual care arm. ${ }^{21}$ Randomisation was, in any case, stratified according to antidepressant use from the outset. We had also assumed that around $65 \%$ of participants receiving usual care would have recovered by the four month follow-up, but results from a trial conducted in a similar population showed that only $20 \%$ recovered. ${ }^{22}$ Finally, our recruitment rate was lower than we expected. The trial steering committee and data monitoring committee therefore approved a recalculation of the sample size required so as to reflect the above changes, resulting in the final sample size of 360 cited previously. Further details are provided elsewhere. ${ }^{65}$

\section{Results}

Sixty five practices agreed to take part in the study, referring a total of 766 patients to the research team. Of 490 people assessed, 361 eligible and consenting participants were successfully randomised (figure $\Downarrow$ ). At baseline, 182 people were allocated to the intervention arm and 179 to the usual care arm. Eighty per cent of the participants provided data at the primary outcome follow-up point. Participants who provided follow-up information on the primary outcome were more likely at baseline to be older, own their own home, be educated to "A" level or beyond, be in receipt of counselling, smoke cigarettes, and drink alcohol less than weekly than those who did not provide such information. Fewer participants were lost to follow-up at the four and 12 month time points, where face to face data collection had taken place.
The randomised groups were similar on entry to the trial, although the proportion of people employed or in education, married or cohabiting, receiving counselling, or not smoking was slightly higher in the intervention group than in the usual care group (table $1 \Downarrow$ ).

\section{Intervention implementation}

The physical activity intervention was delivered according to the manual by all five physical activity facilitators, with allocation of participants to each facilitator based on workload, availability, and location. The number of patients seen by each facilitator ranged from 25 to 58 . Adherence to the intervention was generally good. A mean 7.2 (standard deviation 4.1) sessions was completed, with $56 \%(n=102)$ of participants receiving an adequate dose of at least five contacts by four months post-randomisation. Only $6 \%(n=11)$ of the intervention group failed to attend the first appointment with their physical activity facilitator and $71 \%(n=129)$ had received an adequate dose of intervention by the end of their involvement in the trial.

\section{Primary outcome: short term symptoms of depression}

When the Beck depression inventory score was treated as a continuous variable, there was no evidence that participants in the intervention group had a better outcome at four months than those in the usual care group (table $2 \Downarrow$ ). The intervention group reported slightly lower scores at the four month follow-up, with an adjusted between group difference in mean score of -0.54 (95\% confidence interval -3.06 to $1.99 ; \mathrm{P}=0.68$ ). The proportion of participants who reported a score of less than 10 (that is, "recovered") was slightly smaller in the intervention group at four months post-randomisation $(28.2 \%$ in the intervention arm compared with $35.6 \%$ in the usual care arm), although there was no evidence of a difference between the randomised groups (adjusted odds ratio $0.66,95 \%$ confidence interval 0.4 to 1.11 ; $\mathrm{P}=0.12$ ).

\section{Sensitivity analyses for primary outcome}

Table 2 summarises the results of the additional sensitivity analyses. Firstly, the analysis was performed after multiple imputation of missing data using multiple imputation chained equation methods. The results indicated that missing data might have led to a slight underestimation of the treatment effect, but this was insufficient to alter the overall interpretation of the trial findings. The impact of clustering by practice or by physical activity facilitator was also investigated, but did not alter the results. The calculation of complier-average causal effect estimates indicated that the difference in Beck depression inventory scores between the two treatment groups for those participants who received an "adequate dose" of the intervention was larger than the original intention to treat estimate, although the surrounding confidence interval was wide. Two a priori subgroup analyses provided no evidence to suggest that baseline severity of depression $(\mathrm{P}=0.87)$ or level of physical activity $(\mathrm{P}=0.77)$ had any influence on the difference between intervention and usual care groups.

\section{Secondary outcomes Longer term symptoms of depression}

Table $3 \Downarrow$ presents the results of the repeated measures analysis, in which data from up to three follow-up points were included per participant. There was no evidence of a difference between the treatment groups over the duration of the study (adjusted difference in mean Beck depression inventory score $-1.20,95 \%$ 
confidence interval -3.42 to $1.02 ; \mathrm{P}=0.29)$ or of any differential effect across the three follow-up points (time by treatment interaction $\mathrm{P}=0.61$ ).

\section{Antidepressant use}

The proportion of participants reporting use of antidepressants was smaller in both groups at the end of the trial compared with baseline (table $4 \Downarrow$ ). However, there was no evidence to suggest any difference between the groups at either the four month follow-up point (adjusted odds ratio 1.20, 95\% confidence interval 0.69 to $2.08 ; \mathrm{P}=0.52$ ) or over the duration of the trial. Nor was there any evidence of any differences in treatment effect across the three follow-up points (time by treatment interaction $\mathrm{P}=0.22$ ).

\section{Physical activity}

Table $5 \Downarrow$ shows there was some evidence for a difference in reported physical activity between the groups at four months post-randomisation (adjusted odds ratio 1.58, 0.94 to 2.66; $\mathrm{P}=0.08$ ) and much stronger evidence for this effect when data from all three follow-up points were considered together. However, there was no evidence that the difference between the groups changed over the duration of the study (time by treatment interaction $\mathrm{P}=0.71$ ).

\section{Discussion}

Adults presenting with depressing in primary care and receiving the TREAD (TREAtment of Depression with physical activity) intervention in addition to usual care reported increased physical activity compared with those receiving usual care alone, although there was no evidence to suggest that the intervention brought about any improvement in symptoms of depression or reduction in antidepressant use at the four month follow-up point.

We considered whether our result was sufficiently precise to rule out the possibility of a beneficial effect. The most statistically powerful analysis was using the Beck depression inventory as a continuous outcome measure. The results for our primary analysis indicated an adjusted between group difference in mean Beck depression inventory scores of -0.54 (95\% confidence interval -3.06 to 1.99 ). It is difficult to define precisely what would constitute a clinically important treatment effect, but the National Institute for Health and Clinical Excellence guideline panel ${ }^{23}$ have suggested that this could correspond to around 3 points or 0.35 standard deviations at baseline on the Hamilton depression rating scale ${ }^{24}$ and close to the 0.33 standard deviations used in our power calculation. The equivalent difference in terms of Beck depression inventory score would be between 4.1 and 3.9 points, respectively, based on our observed standard deviation of 11.8 points at four months post-randomisation. This suggests that we have excluded the possibility, at least with $95 \%$ confidence, that the intervention added to usual care is clinically effective in improving symptoms of depression compared with usual care alone.

\section{Strengths and weaknesses of the study}

Our trial recruitment rate was lower than initially planned but we reviewed and amended the variables in our power calculation and subsequently achieved the revised recruitment target. While our retention rate at the four month follow-up was, at $80 \%$, slightly lower than that outlined in the protocol, our sensitivity analyses indicated that missing data probably had little impact on our findings. Moreover, our findings show that the resulting dataset was sufficiently large to estimate confidence intervals that excluded a clinically meaningful difference in outcome between the groups. The trial is one of the largest trials of physical activity and depression to have taken place in primary care. Baseline comparability of the two treatment groups was good. Although we used self reported outcome data in an effort to eliminate any observer bias, it is possible that the participants' responses could have been influenced, to some degree, by their knowledge of their treatment allocation. Physical activity is notoriously difficult to measure and the self reported assessment could have been biased by knowledge of the treatment allocation. The effect of the intervention on physical activity outcomes must therefore be treated with some caution. However, comparison of the data reported in the physical activity recall diary with accelerometry data indicated reasonable agreement between the two measures. ${ }^{6}$ If knowledge of treatment allocation was to bias the responses to physical activity, then it might also be expected to have led to a difference in the scores for symptoms of depression.

\section{Strengths and weaknesses in relation to other studies and important differences in results}

A recent systematic review ${ }^{25}$ of physical activity and depression only included studies of patients with a diagnosis of depression and a follow-up period of more than 16 weeks. Within this restricted analysis there was no longer any evidence for a beneficial effect of physical activity interventions on symptoms of depression. Our results are therefore consistent with that finding, although studies of non-clinical populations over shorter periods suggest a possible benefit. ${ }^{3}$

One important difference between TREAD and previous studies was the development of an intervention that aimed specifically to produce more lasting change in physical activity levels. ${ }^{10}$ Adherence to the intervention supports the perceived acceptability of using the TREAD approach, ${ }^{6}$ and the reported increase in physical activity sustained over the entire 12 month follow-up period indicates that our purpose in designing the intervention was achieved. Indeed the results show a slight increase in the apparent effectiveness of the intervention over the course of the study, which is unusual in trials designed to boost physical activity, with either depressed or non-depressed participants. ${ }^{26}{ }^{27}$ The effect on physical activity reported here (adjusted odds ratio 2.27, 95\% confidence interval 1.32 to 3.89 ) was also larger than the pooled estimate from a recent review of exercise referral schemes (pooled risk ratio 1.16, 95\% confidence interval 1.03 to 1.30$){ }^{27}$ The fact that participants were recruited directly from primary care and were not selected on the basis of their interest or motivation to engage in physical activity adds further weight to the success of the intervention. It is, however, possible that only those participants with relatively positive views of physical activity would have agreed to be involved in the first place.

\section{Meaning of the study, possible explanations, and implications for clinicians and policy makers}

The main implication of our results is that advice and encouragement to increase physical activity is not an effective strategy for reducing symptoms of depression. Although our intervention increased physical activity, the increase may not have been sufficiently large to influence depression outcomes. The absolute difference between the randomised groups in terms of the proportion meeting our physical activity threshold was about $15 \%$ so there is still a possibility that physical activity itself might have some benefits for depression. However, this 
does not alter our conclusion about the lack of effectiveness of facilitated physical activity as an adjunct treatment to usual care for depression. The TREAD intervention was a pragmatic and acceptable intervention that could be implemented in the National Health Service and increase physical activity levels but had no impact on symptoms of depression.

\section{Unanswered questions and future research}

There is empirical evidence from experimental studies in volunteers that some people report improved affect and pleasure while exercising at moderate levels. In contrast, vigorous activity is almost always experienced as unpleasant while it is performed but there is improved affect and pleasure shortly after finishing, perhaps resulting from endogenous opioid activity, the "runner's high." ${ }^{28}$ This might explain why some participants in the trial reported that physical activity helped to improve their mood. ${ }^{29}$ Perhaps an intervention that focused only on vigorous physical activity might be more effective, although we suspect that this would be less acceptable to participants and difficult or even impossible to sustain. A better understanding of the underlying mechanisms that might link physical activity and mood could lead to new therapeutic opportunities.

The TREAD intervention increased self reported physical activity, an effect that lasted beyond the duration of the intervention. Our approach towards increasing physical activity put emphasis on promoting choice and autonomy. It did not rely simply on giving advice but drew on a range of behaviour change techniques. Physical activity might have benefits for patients with medical conditions such as obesity, diabetes, and cardiovascular disease. This approach to increase physical activity might be useful for people with or without depression who also have these other medical conditions.

We thank the patients and staff who participated in the trial; members of our trial steering committee and data monitoring committee for their valuable support throughout the research (Michael King, Chris Dowrick, Margaret Thorogood, Paul Lanham, Helen Lester, Kerry Hood, and Keith Lloyd); the Mental Health Research Network and Primary Care Research Network in connection with data collection; the contribution of the Department of Health and local primary care trusts in meeting the excess treatment and service support costs associated with the trial; and the following colleagues who contributed to the recruitment and retention of patients, provided administrative support to the study, or delivered the intervention to study participants: Georgina Bentley, Susan Bryant, Amanda Burston, Kate Button, Julia Carver, Joy Choules, Sarah Dawkins, Nathan Filer, Davina Chauhan, Emily Gale, Alice Garrood, Charlotte Hale, Caroline Jenkinson, Rhodri Lewis, Linda Mottram, Adam Saint, Sofie Sherlock, Helen Thorp, Tom Thompson, Alison Wellingham, and Joanna Yarham. The views and opinions expressed herein are those of the authors and do not necessarily reflect those of the Department of Health.

Contributors: AMH, NJW, AAM, SPH AHT, KRF, DAL, TJP, DJS, JC, and $G L$ were responsible for the initial proposal, securing funding for the trial, and drafting the original protocol. GL, as principal investigator, had overall management responsibility for the study. MC, as trial coordinator, was responsible for the day to day operationalisation of the trial and monitored data collection. $\mathrm{AMH}, \mathrm{KRF}, \mathrm{AHT}$, and $\mathrm{GL}$ developed the physical activity intervention. All authors contributed to the refinement of the trial protocol and development of the data collections tools. $\mathrm{HB}, \mathrm{RW}, \mathrm{CW}$, and $\mathrm{AS}$ were responsible for data collection and revised the draft paper. MC, NJW, AAM, TJP, DAL, and GL wrote the statistical analysis plan. MC and GL carried out the cleaning, analysis, and interpretation of the data with the assistance of CC, NJW, and AAM. MC and GL wrote the initial draft of the manuscript. All authors contributed to and approved the final manuscript on behalf of the wider research team. MC and CC used data from the study for their PhD and MSc theses, respectively.

Funding: This research was carried out independently by the University of Bristol, University of Exeter and the Peninsula Medical School. It is funded by the Department of Health as part of the National Institute for Health Research Health Technology Assessment programme.

Competing interests: All authors have completed the ICMJE uniform disclosure form at www.icmje.org/coi_disclosure.pdf (available on request from the corresponding author) and declare: no support from any organisation for the submitted work; no financial relationships with any organisations that might have an interest in the submitted work in the previous three years; and no other relationships or activities that could appear to have influenced the submitted work.

Ethical approval: This study was approved by the West Midlands multicentre research ethics committee (MREC 05/MRE07/42), and research governance approval was given by the relevant local National Health Service primary care trusts. The research is listed on the National Research Register and the International Standard Clinical Trial Register. Data sharing: No additional data available.

1 McCrone P, Dhanisiri S, Patel A, Knapp M, Lawton-Smith S. Paying the price: the cost of mental health care in England to 2026. King's Fund, 2008.

2 Hyde J, Calnan M, Prior L, Lewis G, Kessler D, Sharp D. Reluctant medicalisers? A qualitative study exploring how general practitioners decide to prescribe antidepressants. Br J Gen Pract 2005;55:755-62.

3 Mead GE, Morley W, Campbell P, Greig CA, McMurdo M, Lawlor DA. Exercise for depression. Cochrane Database Syst Rev 2009;3:CD004366.

4 National Institute for Health and Clinical Excellence. Depression: the treatment and management of depression in adults (updated edition). British Psychological Society and Royal College of Psychiatrists, 2009.

5 Baxter H, Winder R, Chalder M, Wright C, Sherlock S, Haase A, et al. Physical activity as a treatment for depression: the TREAD randomised trial protocol. Trials 2010;11:105.

6 Chalder M, Wiles N, Campbell J, Hollinghurst S, Searle A, Haase A, et al. A pragmatic randomised controlled trial to evaluate the cost-effectiveness of a physical activity intervention as a treatment for depression: the TREAD trial. Health Technol Assess 2011;16:1-162.

7 Lewis G, Pelosi AJ, Araya R, Dunn G. Measuring psychiatric disorder in the community: a standardised assessment for use by lay interviewers. Psychol Med 1992;22:465-86.

8 Lewis G. Assessing psychiatric disorder with a human interviewer or a computer. $J$ Epidemiol Community Health 1994;48:207-10.

9 Beck AT, Ward CH, Mendelsohn M, Mock J, Erbaugh J. An inventory for measuring depression. Arch Gen Psychiatry 1961;4:561-71.

10 Haase A, Taylor A, Fox KR, Thorp H, Lewis G. Rationale and development of the physical activity counselling intervention for a pragmatic trial of exercise and depression in the UK (TREAD-UK). Ment Health Phys Act 2010;3:85-91.

11 Deci EL, Ryan RM. Intrinsic motivation and self-determination in human behaviour Plenum, 1985

12 Ainsworth BE, Haskell WL, Whitt MC, Irwin ML, Swartz AM, Strath SJ, et al. Compendium of physical activities: an update of activity codes and MET intensities. Med Sci Sports Exerc 2000;32:S498-504.

13 Department of Health Start active, stay active: a report on physical activity from the four home countries' Chief Medical Officers. Department of Health, 2011.

14 Stewart AD, Hays RD, Ware JE. The MOS short-form General Health Survey. Med Care 1988;26:724-32.

15 Resnick B, Zimmerman SI, Orwig D, Furstenberg ALMJ. Outcome expectations for exercise scale: Utility and psychometrics. J Gerontol: Soc Sci 2000;55B:S352-6.

16 Moher D, Hopewell S, Schulz KF, Montori V, Gotzsche PC, Devereaux PJ, et al. CONSORT 2010 explanation and elaboration: updated guidelines for reporting parallel group randomised trials. BMJ 2010;340:c869.

17 StataCorp. Stata Statistical Software. (Release 11). College Station, Texas, 2010.

18 Sterne JA, White IR, Carlin JB, Spratt M, Royston P, Kenward MG, et al. Multiple imputation for missing data in epidemiological and clinical research: potential and pitfalls. $B M J$ 2009;338:b2393.

19 Roberts C, Roberts SA. Design and analysis of clinical trials with clustering effects due to treatment. Clin Trials 2005;2:152-62.

20 Dunn G, Maracy M, Tomenson B. Estimating treatment effects from randomized clinical trials with noncompliance and loss to follow-up: the role of instrumental variable methods. Stat Methods Med Res 2005;14:369-95.

21 Kessler D, Lewis G, Kaur S, Wiles N, King M, Weich S, et al. Therapist-delivered Internet psychotherapy for depression in primary care: a randomised controlled trial. Lancet 2009;374:628-34

22 Lewis G, Mulligan J, Wiles N, Cowen P, Craddock N, lkeda M, et al. Polymorphism of the 5-HT transporter and response to antidepressants: randomised controlled trial. $\mathrm{Br} \mathrm{J}$ Psychiatry 2011;198:464-71.

23 National Institute for Health and Clinical Excellence. Depression: management of depression in primary and secondary care. British Psychological Society and Royal College of Psychiatrists, 2004

24 Hamilton M. A rating scale for depression. J Neurol, Neurosurg Psychiatry 1960;23:56-62.

25 Krogh J, Nordentoft M, Sterne JA, Lawlor DA. The effect of exercise in clinically depressed adults: systematic review and meta-analysis of randomized controlled trials. J Clin Psychiatry 2011;72:529-38.

26 Hillsdon M, Foster C, Thorogood M. Interventions for promoting physical activity. Cochrane Database Syst Rev 2005;1:CD003180. 


\section{What is already known on this topic}

Depression is a leading contributor to disability in the United Kingdom and is associated with a decrement of health greater than many other chronic diseases

Many patients and healthcare professionals would like an effective and accessible non-drug treatment for depression

Numerous studies have reported the positive effects of physical activity but most of the current evidence originates from small non-clinical samples using interventions that are not practicable in healthcare settings

\section{What this study adds}

A physical activity intervention in addition to usual care did not improve symptoms of depression or reduce the use of antidepressants compared with usual care alone

The intervention increased self reported physical activity and this effect was sustained for 12 months

Clinicians and policy makers should alert people with depression that advice to increase physical activity will not increase their chances of recovery from depression

27 Pavey TG, Taylor AH, Fox KR, Hillsdon M, Anokye N, Campbell JL, et al. Effect of exercise referral schemes in primary care on physical activity and improving health outcomes: systematic review and meta-analysis. BMJ 2011;343:d6462.

28 Ekkekakis P, Hall EE, Petruzzello SJ. Variation and homogeneity in affective responses to physical activity of varying intensities: an alternative perspective on dose-response based on evolutionary considerations. J Sports Sci 2005;23:477-500.

29 Searle A, Calnan M, Lewis G, Campbell J, Taylor A, Turner K. Patients' views of physica activity as treatment for depression: a qualitative study. Br J Gen Pract 2011;61:149-56.

Accepted: 22 March 2012

\section{Cite this as: BMJ 2012;344:e2758}

This is an open-access article distributed under the terms of the Creative Commons Attribution Non-commercial License, which permits use, distribution, and reproduction in any medium, provided the original work is properly cited, the use is non commercial and is otherwise in compliance with the license. See: http://creativecommons.org/licenses/bync/2.0/ and http://creativecommons.org/licenses/by-nc/2.0/legalcode. 


\section{Tables}

Table 1| Baseline characteristics of adults with depression randomised to usual care plus facilitated physical activity or to usual care only. Values are numbers (percentages) unless stated otherwise

\begin{tabular}{|c|c|c|}
\hline Characteristics & Intervention group ( $n=182)$ & Usual care group $(n=179)$ \\
\hline Recruited via Bristol centre & $96(53)$ & $96(54)$ \\
\hline Physically active at least one day a week & $92(51)$ & $82(46)$ \\
\hline Mild or moderate depression* & $159(87)$ & $162(91)$ \\
\hline Currently taking antidepressants & $106(58)$ & $101(56)$ \\
\hline Current depressive episode $>6$ months & $90(46)$ & $91(51)$ \\
\hline History of depression & $132(73)$ & $122(68)$ \\
\hline Previously prescribed antidepressants & $117(64)$ & $107(60)$ \\
\hline Immediate family member ever been depressed & $115(63)$ & $114(64)$ \\
\hline Male & $59(32)$ & $63(35)$ \\
\hline White & $170(95)$ & $166(93)$ \\
\hline Married or cohabiting & $91(50)$ & $76(43)$ \\
\hline Currently attending counselling & $38(21)$ & $27(15)$ \\
\hline Employed, studying, or training & $122(67)$ & $132(74)$ \\
\hline Home owner & $87(48)$ & $84(47)$ \\
\hline Educated to A level or beyond & $93(51)$ & $98(55)$ \\
\hline Current smoker & $55(31)$ & $65(37)$ \\
\hline Drinking alcohol at least weekly & $69(39)$ & $72(41)$ \\
\hline At least 1000 MET minutes of physical activity a week & $45(25)$ & $48(28)$ \\
\hline Mean (SD) age at referral & $40.9(12.5)$ & $38.8(12.7)$ \\
\hline Mean (SD) SF-12 standardised physical health score & $51.4(9.8)$ & $50.3(9.9)$ \\
\hline Mean (SD) SF-12 standardised mental health score & $26.5(6.9)$ & $26.8(7.8)$ \\
\hline Mean (SD) Beck depression inventory score & $32.1(9.0)$ & $32.1(9.5)$ \\
\hline Mean (SD) clinical interview schedule-revised score & $28.0(7.9)$ & $28.2(7.8)$ \\
\hline
\end{tabular}

$\mathrm{MET}=$ metabolic equivalent of the task.

*According to clinical interview schedule-revised. 
Table 2| Mean Beck depression inventory (BDI) score and differences in means at four month follow-up of adults with depression allocated to usual care plus facilitated physical activity or to usual care only

\begin{tabular}{|c|c|c|c|c|c|c|c|}
\hline \multirow[b]{2}{*}{ Study arm } & \multirow[b]{2}{*}{ No } & \multirow[b]{2}{*}{$\begin{array}{c}\text { Mean (SD) BDI } \\
\text { score }\end{array}$} & \multicolumn{5}{|c|}{ Difference in means* $(95 \% \mathrm{Cl}), \mathrm{P}$ value } \\
\hline & & & ITT estimate & MICE estimate & $\begin{array}{l}\text { ITT estimate adjusted } \\
\text { for practice clustering }\end{array}$ & $\begin{array}{l}\text { ITT estimate adjusted } \\
\text { for clustering by PAF }\end{array}$ & CACE estimate \\
\hline Intervention & 142 & $16.12(11.34)$ & \multirow{2}{*}{$\begin{array}{c}-0.54(-3.06 \text { to } \\
1.99), 0.68\end{array}$} & \multirow{2}{*}{$\begin{array}{c}-0.76(-3.37 \text { to } \\
1.84), 0.56\end{array}$} & \multirow{2}{*}{$\begin{array}{c}-0.54(-3.06 \text { to } 1.99) \\
0.67\end{array}$} & \multirow{2}{*}{$\begin{array}{c}-0.53(-3.00 \text { to } 1.95) \\
0.68\end{array}$} & \multirow{2}{*}{$\begin{array}{c}-0.86(-4.85 \text { to } \\
3.13), 0.67\end{array}$} \\
\hline Usual care & 146 & $16.87(12.63)$ & & & & & \\
\hline
\end{tabular}

ITT=intention to treat; $\mathrm{MICE}=$ multiple imputation chained equation; $\mathrm{PAF}=$ physical activity facilitator; $\mathrm{CACE}=$ complier-average causal effect.

${ }^{*}$ Adjusted for baseline Beck depression inventory score, antidepressant use, severity of depression, level of physical activity, and recruiting centre. 
Table 3 | Means and differences in mean Beck depression inventory scores at four, eight, and 12 month follow-up in adults with depression allocated to usual care plus facilitated physical activity or to usual care only

\begin{tabular}{|c|c|c|c|c|}
\hline \multirow[b]{2}{*}{ Study arm } & \multicolumn{3}{|c|}{ No (mean score) at follow-up } & \multirow[b]{2}{*}{ Repeated measures difference in means ${ }^{\star}(95 \% \mathrm{Cl}), \mathrm{P}$ value } \\
\hline & 4 months & 8 months & 12 months & \\
\hline Intervention & $142(16.12)$ & $115(14.29)$ & $133(12.59)$ & \multirow[t]{2}{*}{$-1.20(-3.42$ to 1.02$), 0.29$} \\
\hline Usual care & $146(16.87)$ & $107(16.08)$ & $122(13.47)$ & \\
\hline Total No & 288 & 222 & 255 & 308 \\
\hline
\end{tabular}

*Adjusted for baseline antidepressant use, severity of depression, level of physical activity, and recruiting centre. 
Table 4| Percentages and odds ratio of using antidepressants at four, eight, and 12 month follow-up in adults with depression allocated to usual care plus facilitated physical activity or to usual care only

\begin{tabular}{lcccc} 
Study arm & \multicolumn{3}{c}{ No (\%) at follow-up } & \multirow{2}{*}{ Repeated measures odds ratio* (95\% Cl), P value } \\
\cline { 2 - 4 } & $\mathbf{4}$ months & $\mathbf{8}$ months & $\mathbf{1 2}$ months & \\
Intervention & $142(59)$ & $115(42)$ & $133(35)$ & $0.63(0.19$ to 2.06$), 0.44$ \\
\hline Usual care & $147(53)$ & $105(46)$ & $123(42)$ & \\
\hline Total No & 289 & 220 & 256 & 307 \\
\hline
\end{tabular}

*Adjusted for baseline antidepressant use, severity of depression, level of physical activity, and recruiting centre. 
Table 5| Percentages and odds ratio of reporting physical activity ( $\geq 1000$ metabolic equivalent of task minutes a week) at four, eight, and 12 month follow-up in adults with depression allocated to usual care plus facilitated physical activity or to usual care only

\begin{tabular}{lcccc} 
& \multicolumn{3}{c}{ No (\%) at follow-up } \\
\cline { 2 - 4 } Study arm & $\mathbf{4}$ months & $\mathbf{8}$ months & $\mathbf{1 2}$ months & Repeated measures odds ratio* (95\% Cl), P value \\
Intervention & $136(52)$ & $95(63)$ & $123(58)$ & $2.27(1.32$ to 3.89$), 0.003$ \\
\hline Usual care & $136(43)$ & $81(49)$ & $114(40)$ & 293 \\
\hline Total No & 272 & 176 & 237 & 293 \\
\hline
\end{tabular}

*Adjusted for baseline antidepressant use, severity of depression, level of physical activity, and recruiting centre. 


\section{Figure}

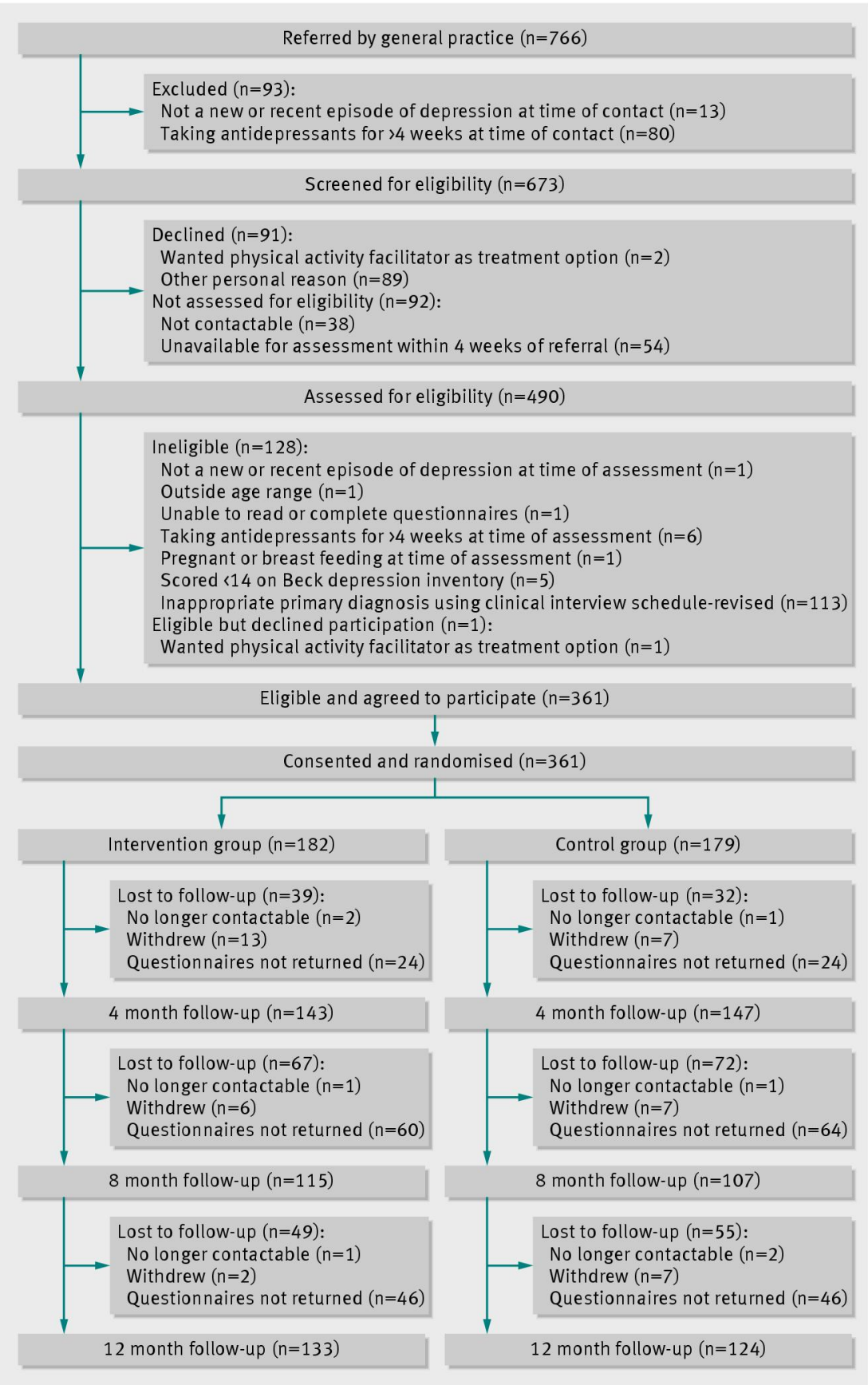

Flow of participants through trial 